\title{
Stereo imaging and X-band radar wave data fusion: An assessment
}

\author{
Benetazzo Alvise ${ }^{1,{ }^{*}}$, Serafino Francesco ${ }^{2}$, Bergamasco Filippo ${ }^{3}$, Ludeno Giovanni ${ }^{4}$, \\ Ardhuin Fabrice ${ }^{6}$, Sutherland Peter ${ }^{5}$, Sclavo Mauro ${ }^{1}$, Barbariol Francesco ${ }^{1}$
}

${ }^{1}$ Institute of Marine Sciences, Italian National Research Council (ISMAR-CNR), Venice, Italy

${ }^{2}$ Institute of Biometeorology, Italian National Research Council (IBIMET-CNR), Florence, Italy

${ }^{3}$ DAIS - Università Ca' Foscari, Venice, Italy

${ }^{4}$ Institute for Electromagnetic Sensing of the Environment, Italian National Research Council (IREA-

CNR), Naples, Italy

${ }^{5}$ Laboratoire d'Océanographie Physique et Spatiale (LOPS), Univ. Brest, CNRS, Ifremer, IRD, Brest,

France

*Corresponding author : Alvise Benetazzo, email address : alvise.benetazzo@ve.ismar.cnr.it

\begin{abstract}
:
The use of spatial and spatio-temporal data is rapidly changing the paradigm of wind wave observations, which have been traditionally restricted to time series from single-point measurements (e.g. from buoys, wave gauges). Active and passive 2D remote sensors mounted on platforms, ships, airplanes and satellites are now becoming standards in the oceanographic community and industry. Given the covered area ranging from centimeters to kilometers, such sensors are now a valuable tool for ocean and coastal observations. In this paper, we intercompare spatio-temporal wind wave data acquired with two state-of-the-art techniques, namely the stereo wave imaging and the X-band marine radar. The comparison was performed by operating the two instruments on an oceanographic research platform during a crossing-sea condition. We analyzed the statistical properties of the wave field, and its directional and omni-directional energy distributions. From our analysis, we suggest that stereo data can be exploited to find the best radar Modulation Transfer Function and scale factor needed to estimate wave parameters. Moreover, the fusion of the two systems will allow to broaden the scales covered by any one measurement, and to retrieve reliable directional wave spectra from short $(\sim 1 \mathrm{~m})$ to midwavelengths $(\sim 100 \mathrm{~m})$.
\end{abstract}

\section{Highlights}

- We compare spatio-temporal wave data from stereo imaging and X-band radar. We use stereo data to improve the radar Modulation Transfer Function (MTF) and scale factor. The fusion of the two systems will allow covering spatial scales broader than any one measurement. 
Keywords: Directional wave observations, Stereo wave imaging, X-band marine radar, Modulation Transfer Function (MTF), Sea technology 


\section{Introduction}

Physical processes associated with the ocean surface wave field are inherently broad-banded, in space as well as time. It is well known that short waves are modulated by long waves (e.g. Cox, 1958; Donelan et al., 2010; Hwang, 2008) and breaking (e.g. Branch and Jessup, 2007), and that the wave growth at a given range of scales is affected by generation and dissipation at other scales (e.g. Kudryavtsev and Chapron, 2016). Moreover, interactions of waves with off-shore structures, bathymetry and coastlines both statistically and in a phase-resolved sense are also highly scale dependent. Therefore, to assess all these processes it is essential to measure the entire distribution of the wave energy, from short to long scales, in space as well as time, and over a broad geographical area, particularly when studying the changes in the wave field as it responds to changes in environmental conditions (e.g. forcing, bathymetry, currents, etc.; Ardhuin et al., 2017).

The vast majority of field measurements of sea surface waves is performed via single-point (1D) temporal observations from buoys (e.g. Holthuijsen, 2008), which provide the omni-directional frequency wave spectrum, and spectral moments that can be related to spectral spreading and directionality (Longuet-Higgins et al., 1963). 2D spatial and 3D spatio-temporal measurements are typically somewhat more difficult to make, but great progress has been made in recent years with techniques like stereo imagery (e.g. Benetazzo et al., 2012; Gallego et al., 2011; Leckler et al., 2015; Benetazzo et al., 2017), X-band marine radar (e.g. Lund et al., 2014; Nieto Borge et al., 2004; Young et al., 1985), polarimetric imaging (e.g. Zappa et al., 2008), airborne scanning lidar (e.g. Hwang et al., 2000; Romero and Melville, 2010; Sutherland and Gascard, 2016), and satellite-borne synthetic aperture radar (Collard et al., 2005; Hasselmann et al., 1985). For measurements of wave breaking, video imagery (e.g Gemmrich et al., 2008; Kleiss and Melville, 2010; Schwendeman and Thomson, 2017) and IR imagery (Sutherland and Melville, 2013) have been used. Roughly, with exact scales depending on the configuration, polarimetry captures short gravity-capillary and gravity waves, stereo imaging captures short- to mid-size wavelengths, X-band radar and scanning lidar capture mid-length to long waves, and synthetic aperture radar captures long waves.

In this paper we focus our attention on two of those instruments, namely the stereo wave imaging and the Xband wave radar, which were operated simultaneously on an oceanographic research platform in the northern Adriatic Sea (Italy). The goal of the study is twofold. On the one hand, we compare the sea surface elevation distribution (including nonlinear parameters) and the directional spectrum to show how well the radar system reproduces the main features of the wave field. Previous studies have already assessed wave radar-derived integral 
wave parameters (e.g Izquierdo et al., 2004) and directional distribution (e.g. with comparison with model data; Lund et al., 2016), but a comparison with stereo systems, which proved to provide accurate 3D wave fields, is still missing. On the other hand, we show that, using stereo data, the empirical radar Modulation Transfer Function (MTF) and scale factor needed to estimate wave parameters can be improved.

This paper is organized as follows. Section 2 gives an overview of wave data and the environmental conditions at the time of their acquisition. The spatio-temporal wave fields and the directional distribution of the energy is analyzed in section 3. In the same section, stereo data are exploited to derive an ad-hoc MTF for wave radar data. In section 4 we draw conclusions of the study and provide an outlook for future use of the two instruments.

\section{Data overview}

In this study, we use a stereo wave imaging system (based on the Wave Acquisition Stereo System, WASS; Benetazzo, 2006; Bergamasco et al., 2017; Benetazzo et al., 2016) and an X-band marine wave radar (hereinafter WR; Ludeno et al., 2014; Serafino et al., 2012) to collect spatio-temporal fields of the sea surface elevation $z$, that is, $z(x, y, t)$, where $\boldsymbol{x}=(x, y)$ denotes the horizontal coordinate vector, and $t$ the time. Instruments were installed on the Acqua Alta oceanographic research platform (Figure 1) in the northern Adriatic Sea (Italy; $45.32^{\circ} \mathrm{N}, 12.51^{\circ} \mathrm{E}$, where the local depth is $17 \mathrm{~m})$.
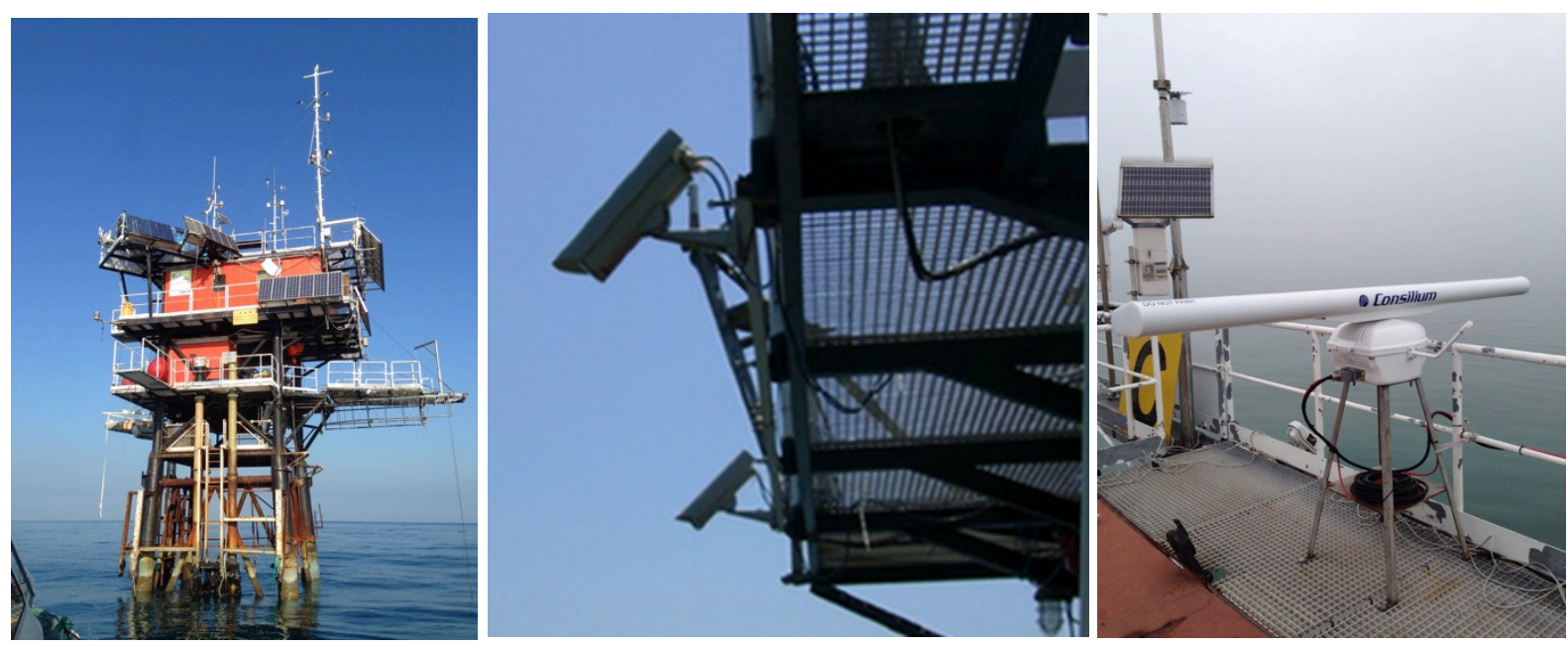

Figure 1 - (left panel) The Acqua Alta oceanographic research platform in the Adriatic Sea (Italy). (middle panel) The WASS deployed at the platform, composed of two synchronized cameras framing a common region of the sea surface. (right panel) The $\mathrm{X}$ band marine radar installed on the platform's roof on the same side as WASS (the left stereo-camera is visible beneath the antenna).

Stereo cameras were deployed on the northeast side of the platform, $2.5 \mathrm{~m}$ apart, and at $12.5 \mathrm{~m}$ height (Figure 1). The system relies on two synchronized digital cameras (with 2456 × 2048 pixels and $3.45-\mu \mathrm{m}$ square active 
elements) mounting 5-mm focal length distortion-less lenses. Image pairs were acquired at $12 \mathrm{~Hz}$, and the sea surface region $\Omega_{\mathrm{S}}$ observed by WASS covers an area $A_{\mathrm{S}}$ of about $3000 \mathrm{~m}^{2}$ (Figure 2). Result of the stereo processing is a temporal sequence of $2 \mathrm{D}$ sea surface elevation fields, that we express for simplicity as $z_{\mathrm{S}}=z_{\mathrm{S}}(x, y$, $t$ ), where $(x, y) \in \Omega_{\mathrm{S}}$. The mapping of $z_{\mathrm{S}}$ on the horizontal mean sea plane was performed following the procedure proposed by Benetazzo (2006), and scatter wave data were linearly interpolated on a spatial grid with uniform $x y$ resolution of $0.2 \mathrm{~m}$. Further details of the stereo pipeline, the post-processing strategy, and the expected accuracy of the $3 \mathrm{D}$ reconstruction (about $3 \mathrm{~cm}$ for the specific configuration) can be found in the studies by Benetazzo et al. (2015) and Bergamasco et al. (2017).

The WR system (Figure 1) is based on a horizontally polarized CONSILIUM X-band radar radiating a maximum power of $25 \mathrm{~kW}$ and equipped with a $2.74 \mathrm{~m}$ long antenna (range resolution of $7 \mathrm{~m}$ ). The radar system is connected to a radar interface, which incorporates an Analog-to-Digital converter for the received signal. The radar images are stored on a $1024 \times 1024$ cells Cartesian grid. As is typical for conventional WRs, the measured backscatter intensities were not radiometrically calibrated. The radar field of view $\Omega_{\mathrm{R}}$ spans $270^{\circ}$ and bounds a sea surface region with area $A_{\mathrm{R}} \sim 16 \mathrm{~km}^{2}$ (range up to $\approx 2620 \mathrm{~m}$ ), which was sampled with a grid of 5.13-m uniform resolution on the horizontal $x y$-plane. The antenna rotation period is $1.87 \mathrm{~s}$.

The backscatter data from the WR were analyzed with a well-established standard algorithm (Nieto Borge et al., 2004; Young et al., 1985) based on sequential steps in the 3D spectral energy domain $\left(k_{\mathrm{x}}, k_{\mathrm{y}}, f\right)$, where $\boldsymbol{k}=$ $\left(k_{\mathrm{x}}, k_{\mathrm{y}}\right)$ is the wavenumber vector, and $f$ is the frequency. After high-pass and band-pass filtering the backscatter in the 3D spectral domain and correcting for the sea current-induced Doppler effect (Huang et al., 2016; Senet et al., 2001; Serafino et al., 2010), to account for the radar image formation nonlinear mechanisms, an empirical omnidirectional MTF proportional to $k^{1.2}$ was applied to the variance of each sea clutter. In doing this, it is assumed negligible the variability of hydrodynamic modulation, tilt modulation and shadowing with the range and azimuth, as well as the effect on the phases of each spectral mode (Nieto Borge et al., 2004). Then, the record of non-scaled sea surface elevation $z_{\mathrm{R}}^{*}=z_{\mathrm{R}}^{*}(x, y, t)$, with $(x, y) \in \Omega_{\mathrm{R}}$, is recovered by inverting back from the wavenumberfrequency spectral to the spatio-temporal domain (Figure 2).

At Acqua Alta, wave data were acquired with WASS and WR on 27 March 2014, starting both acquisitions at 09:10 UTC. WASS operated for 30 minutes, while the WR recorded backscatter data for about $240 \mathrm{~s}$. The local wind and wave conditions were provided by in-situ reference instrumentation (a Nortek Acoustic Wave and 
Current profiler, AWAC, for waves, and a VT0705B SIAP anemometer for wind parameters), which gives the picture of a local northeasterly wind (mean direction of $56^{\circ} \mathrm{N}$ ) blowing with a mean speed (at $17.5 \mathrm{~m}$ height) of $10.6 \mathrm{~m} / \mathrm{s}$. Locally, wind generated waves were in a fetch-limited condition with total spectral significant wave height $H_{\mathrm{m} 0}=1.38 \mathrm{~m}$, peak period $T_{\mathrm{p}}=4.9 \mathrm{~s}$, and peak direction of wave propagation $\theta_{\mathrm{p}}=257^{\circ} \mathrm{N}$. The sea current, measured by the same instrument AWAC, was almost uniform along the vertical direction (from the surface to the sea bottom), with a mean speed of $0.25 \mathrm{~m} / \mathrm{s}$ and mean direction of propagation around $230^{\circ} \mathrm{N}$. The wave condition was more complex than what synthetic spectral parameters can tell, as we observed early on 27 March 2014 surface winds rotating southeast about $300 \mathrm{~km}$ from the Acqua Alta platform at the scale of the Adriatic Sea basin (e.g. http://www.isac.cnr.it/dinamica/projects/forecasts/moloch/). This meteorological pattern influences the wave energy directional distribution, as we shall show in next sections using WASS and WR measurements.
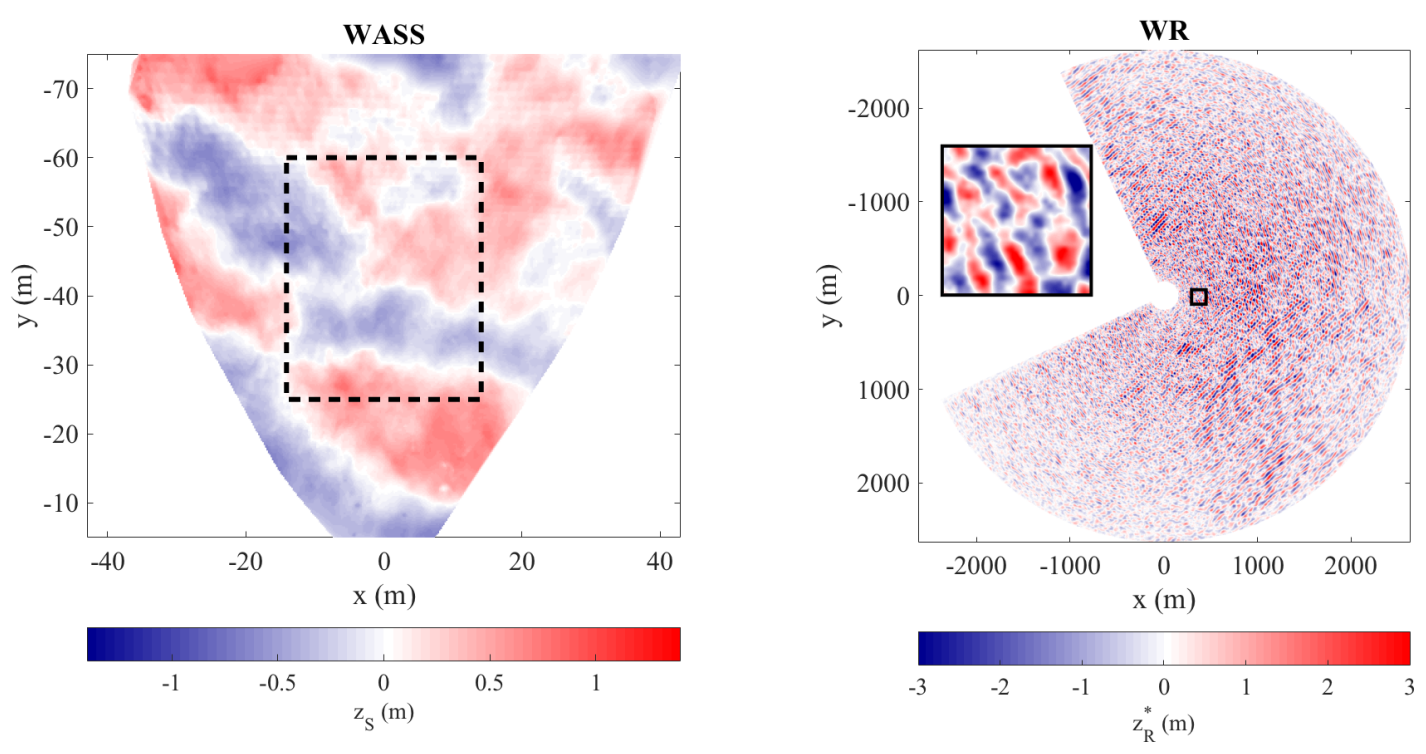

Figure 2 - (left panel) Example of 2D wave field $z_{S}=z_{S}(x, y)$ measured by WASS. The dashed black rectangle frames the window (of area $28.0 \times 34.8 \mathrm{~m}^{2}$ ) used for the 3D spectral analysis. WASS data are shown in the camera reference system, whose $y$-axis is rotated clockwise $226^{\circ}$ wrt to North. (right panel) Example of 2D non-scaled wave field $z_{\mathbf{R}}^{*}(x, y)$ acquired with the WR. The field within the black square $\left(159 \times 159 \mathrm{~m}^{2}\right)$ is magnified on the left-hand side of the panel. WR data are shown in the geographical reference system. On both panels, the origin $(x, y)=(0 \mathrm{~m}, 0 \mathrm{~m})$ is placed on the instrumental center (left stereo-camera principal point and radar antenna midpoint).

\section{Analysis and results}

In this section, we analyze $2 \mathrm{D}+$ time wave data collected with the two instruments WASS and WR, pursuing the intercomparison of their performances in providing the directional and omni-directional distribution of the wave 
energy. A necessary condition for a meaningful analysis is that the elevation field is statistically homogeneous in space and stationary in the time interval considered (e.g. the duration of the record should be shorter than about 30 minutes; Holthuijsen, 2008). The condition of homogeneity is generally fulfilled by stereo measurements that are able to observe a sea surface region with area $\sim 10^{3}-10^{4} \mathrm{~m}^{2}$. For the installation on the Acqua Alta platform, such a requirement is also met by the WR, as the local bathymetry is gently sloping and the instrumental range is relatively small compared to the scales of the local variability of the wave field (e.g. Benetazzo et al., 2013).

Apart from the environmental variability, the WR's dependence on range and azimuth is a complex function of the radar backscatter from the sea surface (Lund et al., 2014). Figure 3 gives an example of this dependence showing, for each location of the $x y$-plane, the standard deviation $\sigma$ of the time series of non-scaled sea surface elevations $z_{\mathrm{R}}^{*}(t)$. We observe that the power of the backscatter signal decays super-linearly with the range (Gommenginger et al., 2000). However, since our purpose is not to investigate the dependence on range and azimuth of the WR strength of the surface wave signal, for the comparison between WASS and WR, we have selected a single analysis window (with area of $1031 \times 1031 \mathrm{~m}^{2}$, and shown as dashed black square in Figure 3 ) in the high-energy near-range of the WR, within which, however, we still observe a variability of the local wave variance by a factor of 2-3. This variability was well accounted for in the recent study by Qiu et al. (2017), who proposed a range- and azimuth-dependent MTF, which, however, was not adopted in our study.

It is worth noting that the non-overlapping sea surface regions covered by WASS and the WR do not permit a wave-to-wave comparison of the observations, but only a phase-averaged assessment of the energy distribution and statistical properties, assuming, however, the analysis area of the two instruments representative of the same (in a statistical sense) sea state. This assumption will allow to obtain straightforwardly an approximation of the WR actual sea surface elevation $z_{\mathrm{R}}=z_{\mathrm{R}}(x, y, t)$, scaling $z_{\mathrm{R}}^{*}(x, y, t)$ with the non-dimensional ratio $\gamma=$ $\sigma\left\{z_{\mathrm{S}}\right\} / \sigma\left\{z_{\mathrm{R}}^{*}\right\}$ between the standard deviation of the stereo field $z_{\mathrm{S}}, \sigma\left\{z_{\mathrm{S}}\right\}=0.34 \mathrm{~m}$, and of $z_{\mathrm{R}}^{*}, \sigma\left\{z_{\mathrm{R}}^{*}\right\}$. For the WR signal within the analysis window, we estimated $\gamma=0.28$. We note that, for WASS, the significant wave height estimated as $H_{\mathrm{s}}=4 \sigma\left\{z_{\mathrm{S}}\right\}=1.36 \mathrm{~m}$ agrees well (difference of $2 \mathrm{~cm}$ ) with the value provided by the reference instrumentation. 


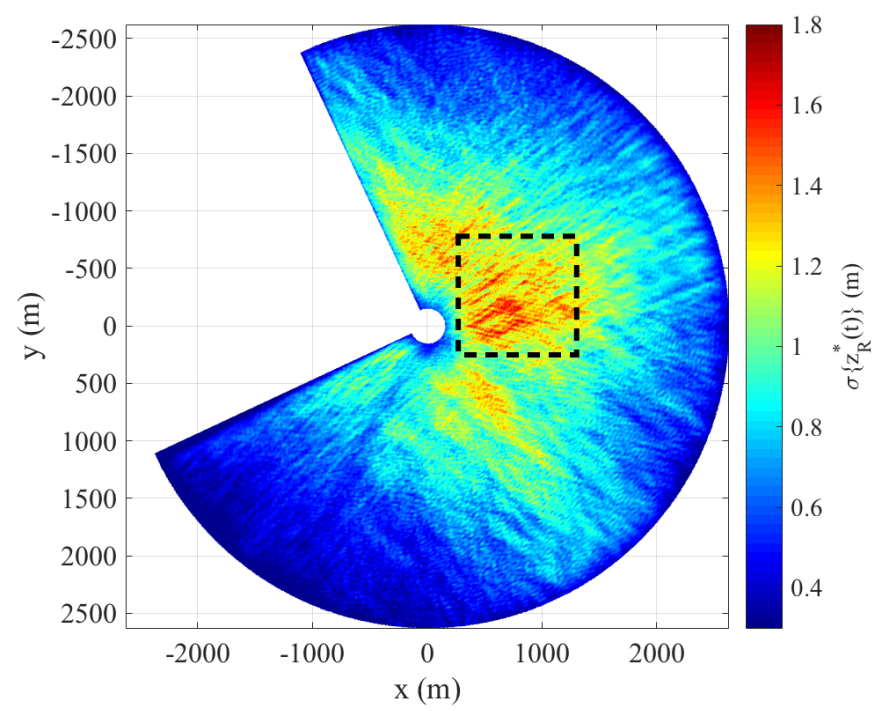

Figure 3 - WR strength of the surface wave signal. Map of the standard deviation $\sigma$ of the time record $z_{\mathrm{R}}^{*}(t)$ at each grid cell of the $x y$-plane. The dashed black square frames the analysis window, located in the near-range of the field of view. Data are shown in the geographical reference system.

\subsection{Directional wave spectrum and nonlinear effects}

The 3D variance density spectrum of the sea surface elevation field $z(x, y, t)$ is interpreted as the distribution of the wave energy $S$ over wavenumbers, and frequencies, that is, $S\left(k_{\mathrm{x}}, k_{\mathrm{y}}, f\right)$. The $3 \mathrm{D}$ wave spectrum is conventionally computed via discrete Fourier Transform (hereinafter FT) of the spatio-temporal record $z(x, y, t)$. During windforced wave conditions, the 3D spectrum shows two characteristic modes (see Figure 4 and, for example, Figure 3 in Peureux et al., 2017), associated with the variance of free and nonlinear bound waves (e.g. Janssen, 2009). In the $\boldsymbol{k} f$-space, the distribution of free modes closely follows the dispersion relation for linear waves, even though a nonnegligible contribution is given by nonlinear wave-wave interactions that redistribute energy among the free wave components. Although bound modes are less energetic than free modes (Longuet-Higgins, 1963), the former have a fundamental role in shaping real waves, producing waves with high sharp crests and shallow rounded troughs (Figure 5). The contribution of bound harmonics manifests in an excess kurtosis $\left(m_{4}-3\right)$ and non-zero skewness $\left(m_{3}\right)$ of the distribution of sea surface elevation (Longuet-Higgins, 1963). These two parameters are estimated from each 3D field $z=z(x, y, t)$ using the following formulae:

$$
m_{3}=<\left(\frac{z-\mu}{\sigma}\right)^{3}>, m_{4}=<\left(\frac{z-\mu}{\sigma}\right)^{4}>
$$

where $\mu=<z>$ and the angle brackets $<>$ denote the ensemble average. 


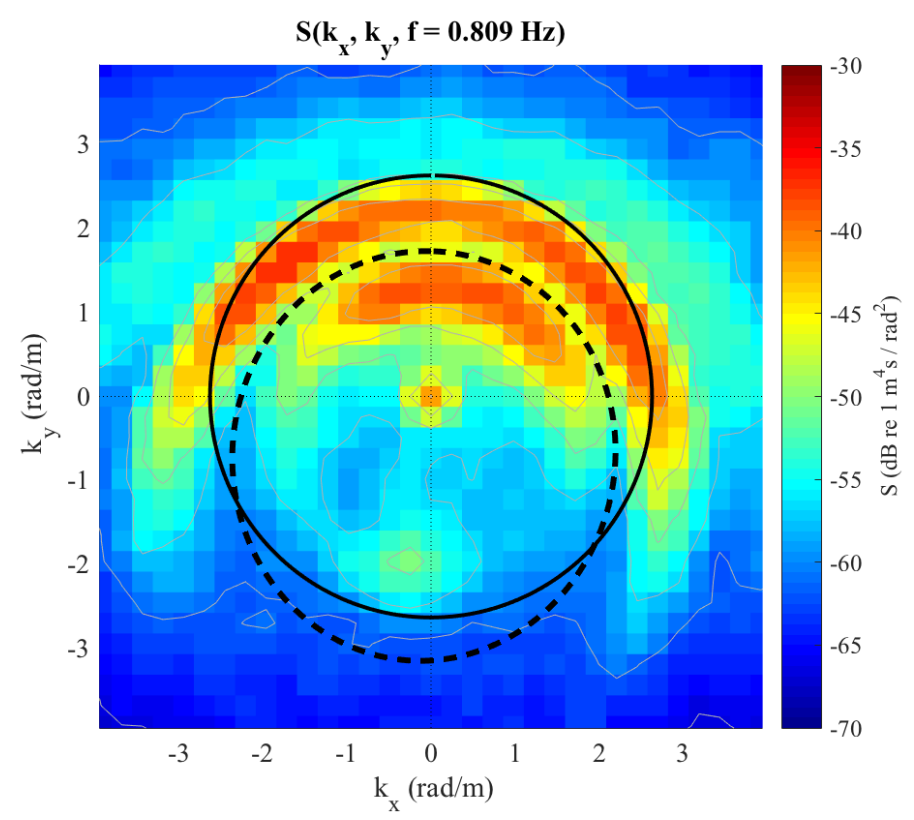

Figure 4 - 2D section of the 3D spectrum $S\left(k_{\mathrm{x}}, k_{\mathrm{y}}, f\right)$ at given frequency $f=0.809 \mathrm{~Hz}$ (the energy appears in the direction where it is coming from). The wave spectrum is computed via FT of the WASS wave field $z_{S}(x, y, t)$ and it is shown in the camera reference system. The black dashed line correspond to $k^{*}=0.83 f^{2} / g$ (Leckler et al., 2015) where $g$ is the acceleration caused by the gravity force; $k^{*}$ separates the free modes (outside the line) from the positive interaction terms of bound modes (inside the line). Note that, in the $k_{\mathrm{x}} k_{\mathrm{y}}$-plane, the distribution of wave energies is apparently distorted by the presence of a sea current (the black solid line shows the trace of the linear dispersion relation in still waters), and the distribution of free modes is bimodal around the peak direction.

The standard procedure used to remove background noise from the WR backscatter data adopts a bandpass filter in the Fourier space around the linear dispersion relation for gravity waves. This operation preserves most of the energy of the sea state providing realistic wave spectra. The effect of the band-pass filter on the WR field $z_{\mathrm{R}}^{*}$ is visible in Figure 5 (right panel). In fact, it is observed that the WR data distribution is symmetric around the mean value (the skewness coefficient $m_{3}=0$ ) and closely follows the Gaussian distribution (even though slightly more peaked, the kurtosis coefficient being $m_{4}=2.91<3$ ). For WASS data, we verify an agreement between the empirical histogram and the nonlinear theoretical probability density function (GC4-WASS in Figure 5; Longuet-Higgins, 1963) that accounts for the coefficients of skewness $\left(m_{3}=0.16\right)$ and kurtosis $\left(m_{4}=3.14\right)$. 

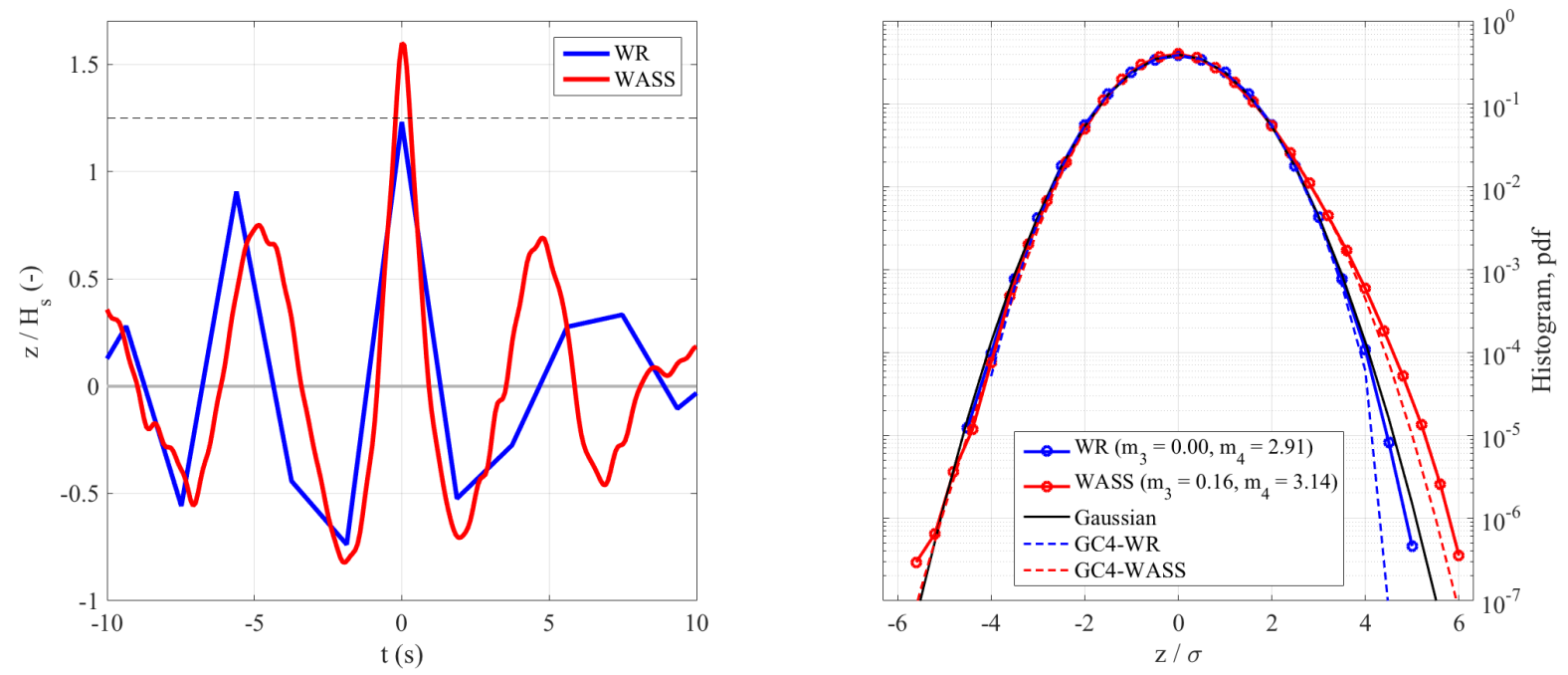

Figure 5 - (left panel) Temporal profile of the normalized sea surface elevation around the maximum crest height, max $\{z(x, y, t)\}$, for the MR and WASS spatio-temporal wave fields. The horizontal dashed gray line at 1.25 represents the rogue wave threshold. (right panel) Histogram of the normalized sea surface elevation field $z$. WASS and WR empirical distributions are compared with the Gaussian probability density function (pdf) and its nonlinear correction (GC4) that accounts for the coefficients of skewness ( $m_{3}$ ) and kurtosis $\left(m_{4}\right)$.

Further, the 2D wavenumber spectrum $S\left(k_{\mathrm{x}}, k_{\mathrm{y}}\right)$ is obtained by integrating the 3D spectrum $S\left(k_{\mathrm{x}}, k_{\mathrm{y}}, f\right)$ with respect to the frequencies as follows:

$$
S\left(k_{\mathrm{x}}, k_{\mathrm{y}}\right)=\int S\left(k_{\mathrm{x}}, k_{\mathrm{y}}, f\right) \mathrm{d} f
$$

The spectrum $S\left(k_{\mathrm{x}}, k_{\mathrm{y}}\right)$ is related to the wavenumber/direction $S(k, \theta)$ spectrum in polar coordinates by the following relationship:

$$
S(k, \theta)=S\left(k_{\mathrm{x}}, k_{\mathrm{y}}\right) J
$$

where $J=k$ is the Jacobian used to transform the spectrum from the Cartesian $\left(k_{\mathrm{x}}, k_{\mathrm{y}}\right)$-domain to the polar $(k, \theta)$ domain. However, the discrete wavenumber-frequency FT is folded over a specific spectral range, which depends on the size and resolution of the spatio-temporal domain. As a consequence, typical stereo observations provide low directional resolution of the spectrum at frequencies smaller than about $0.2 \mathrm{~Hz}$ (e.g. Peureux et al., 2017), which limits the overlap between stereo and radar data. To solve the problem, alternative decompositions for stereo observations, like stochastic methods (e.g. EMEP; Hashimoto et al., 1994) or the Wavelet Directional Method (Donelan et al., 1996), can provide reliable directional spectra $S(f, \theta)$ and $S(k, \theta)$ over a wide range of wavenumbers and frequencies. 
The directional spectrum $S(k, \theta)$ and distribution $D(\theta)$ computed using WR data (via FT) and WASS data (via EMEP) are shown in Figure 6 and Figure 7, respectively. Spectra are consistent to show a bi-modal sea condition, with two distinct energy peaks. The dominant one is located at $250{ }^{\circ} \mathrm{N}$ (WR) and $258^{\circ} \mathrm{N}$ (WASS), and the secondary one at $310^{\circ} \mathrm{N}(\mathrm{WR})$ and $320^{\circ} \mathrm{N}$ (WASS). The most energetic wave component (at $k \approx 0.16 \mathrm{rad} / \mathrm{m}$ for both WR and WASS, consistent with the AWAC observation) was forced by the local northeasterly wind condition, while the least one (a swell with peak at $k \approx 0.11 \mathrm{rad} / \mathrm{m}$ ) was produced by southeasterly winds blowing in the far field (central Adriatic Sea). There is a small difference between directions of the peaks provided by the two instruments, and it is interesting that both WASS and WR well accomplish to detect the larger directional spreading expected for the wind waves compared to the swell (Forristall and Ewans, 1998). We note that the two distributions $D(\theta)$, albeit highly correlated (the cross-correlation coefficient between the two series is 0.88 ), show an offset of $8^{\circ}$, which we attribute to a misalignment between the two instruments (after correction of the offset the cross-correlation coefficient is 0.91 ).
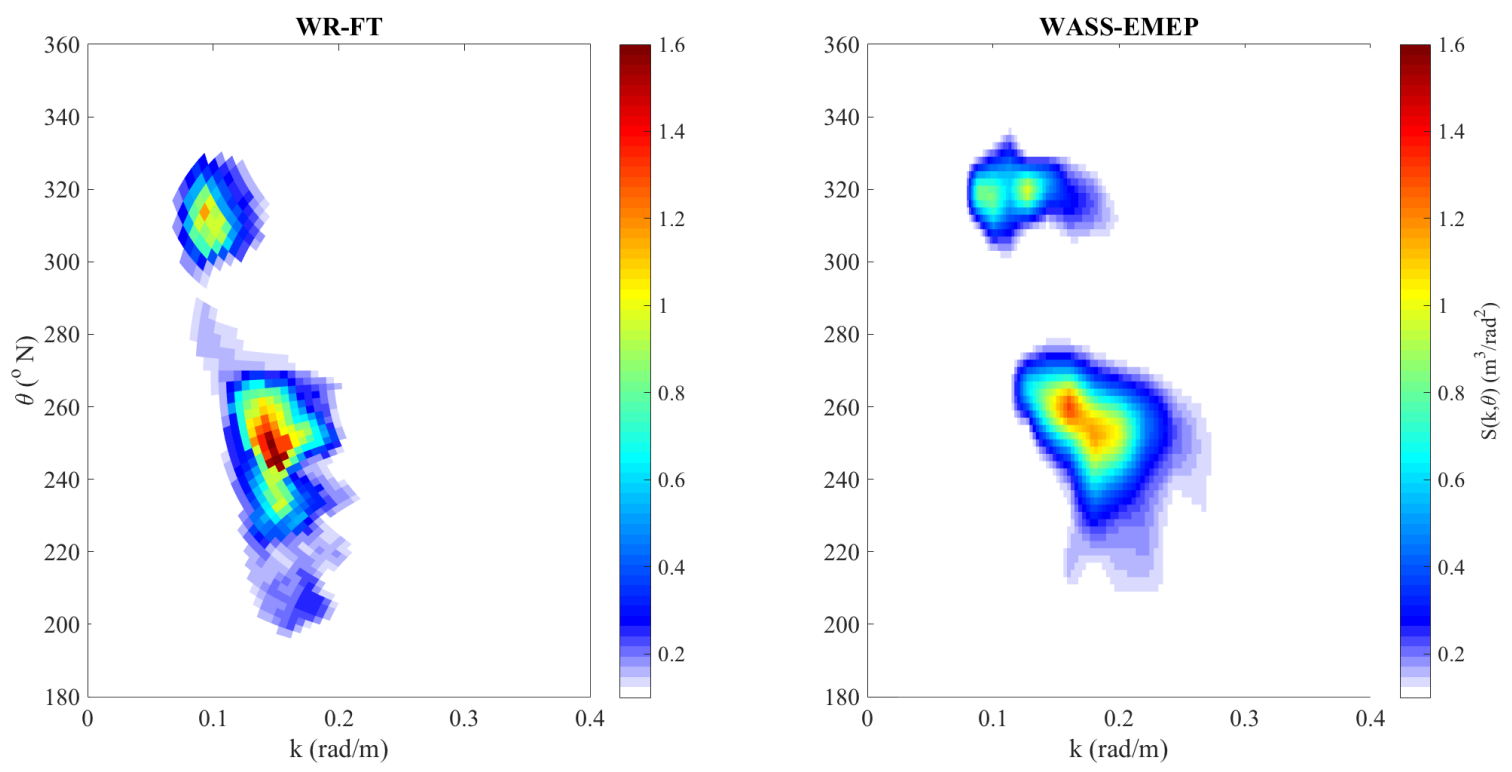

Figure 6 - (left panel) WR wavenumber-direction spectrum obtained from $S\left(k_{\mathrm{x}}, k_{\mathrm{y}}, f\right)$ integrating over all frequencies (we use flow direction of the wave energy). The energy is scaled to match the total variance of the stereo field $z_{\mathrm{S}}$. The wavenumber domain ranges from $-0.6124 \mathrm{rad} / \mathrm{m}$ to $0.6124 \mathrm{rad} / \mathrm{m}$ with a resolution of $0.0061 \mathrm{rad} / \mathrm{m}$. For graphical purposes, a smoothing is performed for each spectral element by averaging in a 5-by-5 neighborhood. (right panel) WASS spectrum $S(k, \theta)$ computed converting the EMEP spectrum $S(f, \theta)$ using the linear dispersion relation for gravity waves. The wave spectrum is resolved with 180 equally spaced directions to cover the full circle and 1024 equally distributed frequencies in the range 0.05 to $2.00 \mathrm{~Hz}$. 


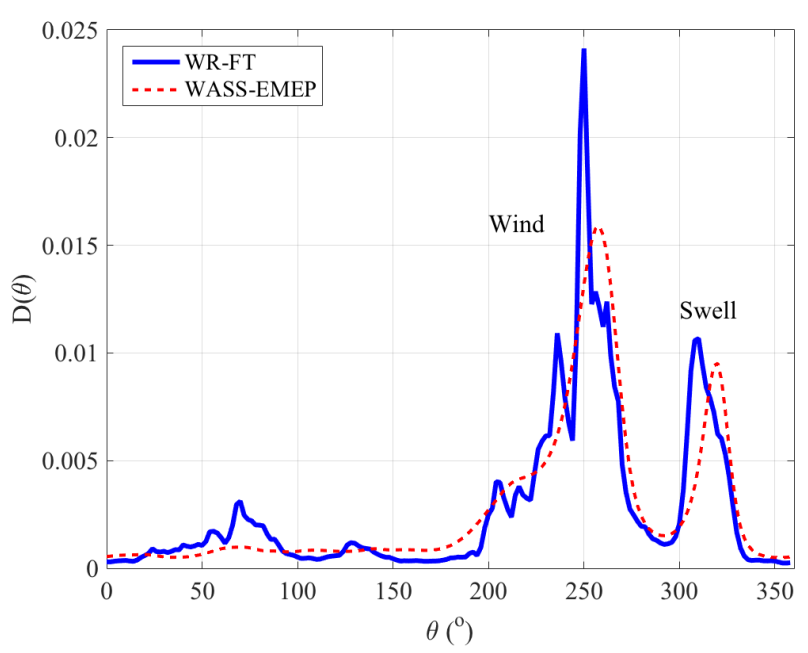

Figure 7 - Omni-wavenumber directional distribution $D(\theta)$ of the spectrum $S(k, \theta)$ in Figure 6. The two wave systems are labeled as "Wind" and "Swell".

\subsection{Omni-directional wavenumber spectrum}

Since the radar image spectrum is converted to the wave spectrum with a MTF proportional to a power of the wavenumber $k$, it is convenient to compare the omni-directional wavenumber spectra $S(k)$ computed using the WASS and WR wave fields. For WASS, two different $S(k)$ are provided, the first one (WASS-EMEP) obtained integrating over all directions the EMEP-derived spectrum $S(k, \theta)$, and the second one (WASS-FT) computed via FT of the 3D stereo field $z_{\mathrm{S}}$ within the analysis window shown in Figure 2. The latter, however, has a small spectral resolution (the minimum wavenumber is about $0.2 \mathrm{rad} / \mathrm{m}$ ), and it is used here with the main purpose of assessing the output from EMEP. Results are presented in Figure 8, where the radar spectrum (WR-FT) is computed via FT of the 3D field $z_{\mathrm{R}}^{*}$. 


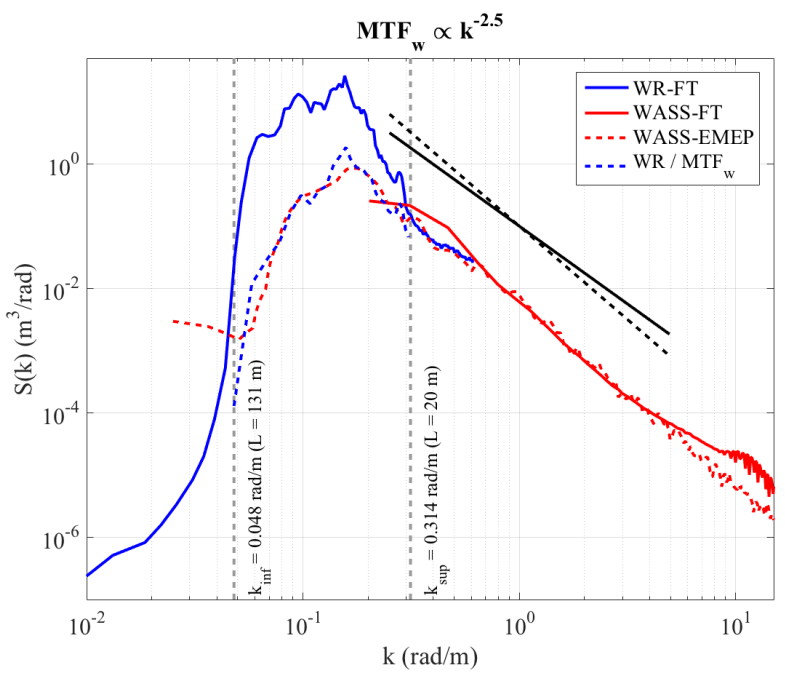

Figure 8 - Omni-directional wavenumber spectrum $S(k)$. WR-FT: WR spectrum computed using the image inversion algorithm of Nieto Borge et al. (2004). WASS-FT: WASS spectrum computed using the FT of the 3D stereo field $z_{\mathrm{S}}$. WASS-EMEP: WASS spectrum computed from $S(k, \theta)$ obtained using EMEP. WR/MTF w $_{\mathrm{w}}$ WR spectrum determined applying the Modulation Transfer Function $\mathrm{MTF}_{\mathrm{w}}$. The black solid and dashed lines are reference spectral slopes proportional to $k^{-5 / 2}$ and $k^{-3}$, respectively. Vertical gray lines show the two limits $\boldsymbol{k}_{\text {inf }}$ and $\boldsymbol{k}_{\text {sup }}$ for WR-FT.

Albeit WASS and WR spectral domains have different sizes, omni-directional spectra $S(k)$ share a common range of wavenumbers and have interesting features that we describe in detail. As far as the WR is concerned, the high-pass filter of the image spectra results in a drop of energy level around $k=k_{\text {inf }}=0.048 \mathrm{rad} / \mathrm{m}(\mathrm{which}$ corresponds to filter out long wave components with length $L$ greater than $131 \mathrm{~m}$ ), while a change in the spectral slope is seen above $k=k_{\text {sup }}=0.314 \mathrm{rad} / \mathrm{m}$, corresponding to $L=20 \mathrm{~m}$, i.e. about four times the resolution ( $5.13 \mathrm{~m}$ ) of the gridded radar data. We note that for WR-FT the energy decays, above the response peak, more rapidly than $k^{-5 / 2}$, that is the typical slope of the Kolmogorov-type energy cascade caused by the resonant four wave-interactions (Zakharov and Filonenko, 1967), or of the model of the equilibrium range (Phillips, 1985). The distortion of the high-wavenumber spectral slope for WRs was also observed by Ludeno et al. (2014), who proposed an adjustment of the MTF by Nieto Borge et al. (2004) to provide more accurate estimations of the spectral shape. On the contrary, as it has been already proved in many studies (e.g. Benetazzo et al., 2012), stereo observations correctly reproduce the slope in the equilibrium range up to wavenumbers around $\sim 10 \mathrm{rad} / \mathrm{m}$ (Peureux et al., 2017), as it is confirmed by both WASS-FT and WASS-EMEP spectral shapes. Indeed, for WASS-EMEP, the significant wave height $H_{\mathrm{m} 0 \text {,wASs }}$ (computed as four times the zero-th order moment of the spectrum) is $1.40 \mathrm{~m}$, which well agrees with the observation from AWAC. 
Hence, using WASS-EMEP as a reference, a least-square fitting (with the same procedure as in Nieto Borge et al., 2004) between WR-FT and WASS-EMEP in the range $\left[k_{\text {inf }}, k_{\text {sup }}\right]$ can provide the function $\left(\mathrm{MTF}_{\mathrm{w}}\right)$ to be applied to the radar spectrum to reconcile with the stereo spectrum. For our specific case, we have estimated an

$\mathrm{MTF}_{\mathrm{w}} \propto k^{-2.5}$ that provides also the scaling of the energy of the WR spectrum, from which we obtain a calibrated estimation of the radar field. This can be verified by observing that, after applying $\mathrm{MTF}_{\mathrm{w}}$, the WR significant wave height becomes $H_{\mathrm{m} 0, \mathrm{wR}}=1.36 \mathrm{~m}$.

\section{Conclusions and outlook}

In this study, we have made for the first time a comparison between directional and omni-directional spectra retrieved by two widely used oceanographic instruments that provide spatio-temporal wave data, that is, a marine radar and a stereo wave imaging system. Albeit able of observing partially overlapping portions of the wavenumber-frequency spectrum, the WR and WASS can be used to accurately establish the directional properties of the wave field, which we have verified are consistent between the two instruments. In general, while stereo methods proved to be more accurate in the reconstruction of 3D wave fields, the larger spatial coverage provided by the X-band radar allows a wider region of the sea surface to be explored.

Our analysis supports the conclusion that it is convenient to simultaneously operate the WR and the WASS, at least during the calibration stages of the WR. Indeed, as the WR linearized wave spectra and wave parameters exhibit a strong dependency on analysis window range and azimuth, an ad-hoc MTF and scale factor can be determined using WASS. Moreover, the fusion between the two instruments will lead to measurements of wave spectra covering ranges broader than those provided by each instrument, with the possibility to retrieve reliable wave energy levels from short $(\sim 1 \mathrm{~m})$ to mid-length $(\sim 100 \mathrm{~m})$ scales. The prospect for future investigations should include the comparison with wave buoys, with the main purpose of assessing the two-dimensional distribution of energy obtained by those instruments, which provide the vast majority of wave measurements across the world's oceans.

\section{Acknowledgements}

Authors gratefully acknowledge the funding from the Flagship Project RITMARE - The Italian Research for the Sea - coordinated by the Italian National Research Council and funded by the Italian Ministry of Education, 
University and Research within the National Research Program 2011-15. The authors acknowledge the support from the project WAVESCALE (LabexMER). Dr. Mauro Bastianini (ISMAR-CNR) and Dr. Mauro Manente are thanked for assistance during deployments at Acqua Alta. The stereo pipeline used to calibrate stereo cameras and to process images collected at Acqua Alta is available as open-source software at http://www.dais.unive.it/wass/.

\section{References}

Ardhuin, F., Gille, S.T., Menemenlis, D., Rocha, C.B., Rascle, N., Chapron, B., Gula, J., Molemaker, J., 2017. Small-scale open ocean currents have large effects on wind wave heights. J. Geophys. Res. O 122, 45004517. doi:10.1002/2016JC012413.Received

Banner, M.L., Jones, I.S.F., Trinder, J.C., 1989. Wavenumber spectra of short gravity waves. J. Fluid Mech. 198, $321-344$.

Benetazzo, A., 2006. Measurements of short water waves using stereo matched image sequences. Coast. Eng. 53, 1013-1032. doi:10.1016/j.coastaleng.2006.06.012

Benetazzo, A., Ardhuin, F., Bergamasco, F., Cavaleri, L., Veras, P., Schwendeman, M., Sclavo, M., Thomson, J., Torsello, A., 2017. On the shape and likelihood of oceanic rogue waves. Sci. Rep. 7, 1-11. doi:10.1038/s41598-017-07704-9

Benetazzo, A., Barbariol, F., Bergamasco, F., Torsello, A., Carniel, S., Sclavo, M., 2016. Stereo wave imaging from a moving vessel: practical use and applications. Coast. Eng. 109, 114-127. doi:10.1016/j.coastaleng.2015.12.008

Benetazzo, A., Barbariol, F., Bergamasco, F., Torsello, A., Carniel, S., Sclavo, M., 2015. Observation of extreme sea waves in a space-time ensemble. J. Phys. Oceanogr. 45, 2261-2275. doi:10.1175/JPO-D-15-0017.1

Benetazzo, A., Carniel, S., Sclavo, M., Bergamasco, A., 2013. Wave-current interaction: Effect on the wave field in a semi-enclosed basin. Ocean Model. 70, 152-165. doi:10.1016/j.ocemod.2012.12.009

Benetazzo, A., Fedele, F., Gallego, G., Shih, P.-C., Yezzi, A., 2012. Offshore stereo measurements of gravity waves. Coast. Eng. 64, 127-138. doi:10.1016/j.coastaleng.2012.01.007

Bergamasco, F., Torsello, A., Barbariol, F., Sclavo, M., Benetazzo, A., 2017. WASS: an Open-Source Pipeline for 3D Stereo Reconstruction of Ocean Waves. Comput. Geosci. doi:10.1016/j.cageo.2017.07.001

Branch, R., Jessup, A.T., 2007. Infrared Signatures of Microbreaking Wave Modulation. IEEE Geosci. Remote Sens. Lett. 4, 372-376.

Collard, F., Ardhuin, F., Chapron, B., 2005. Extraction of Coastal Ocean Wave Fields From SAR Images. IEEE J. Ocean. enginnering 30, 526-533.

Cox, C.S., 1958. Measurements of slopes of high-frequency wind waves. J. Mar. Res. 16, 199-225.

Donelan, M., Drennan, W.M., Magnusson, A.K., 1996. Nonstationary analysis of the directional properties of propagating waves. J. Phys. Oceanogr. 26, 1901-1914.

Donelan, M.A., Haus, B.K., Plant, W.J., Troianowski, O., 2010. Modulation of short wind waves by long waves. J. Geophys. Res. 115, 1-12. doi:10.1029/2009JC005794

Forristall, G.Z., Ewans, 1998. Worldwide Measurements of Directional Wave Spreading. J. Atmos. Ocean. 
Technol. 15, 440-469.

Gallego, G., Yezzi, A., Fedele, F., Benetazzo, A., 2011. A variational stereo method for the three-dimensional reconstruction of ocean waves. IEEE Trans. Geosci. Remote Sens. 49. doi:10.1109/TGRS.2011.2150230

Gemmrich, J., Banner, M.L., Garrett, C., 2008. Spectrally Resolved Energy Dissipation Rate and Momentum Flux of Breaking Waves. J. Phys. Oceanogr. 38, 1296-1312. doi:10.1175/2007JPO3762.1

Gommenginger, C..-, Ward, N.P., Fisher, G.J., Robinson, I.S., Boxall, S., 2000. Quantitative microwave backscatter measurements from the ocean surface using digital marine radar images. J. Atmos. Ocean. Technol. 17, 665-678.

Hashimoto, N., Nagai, T., Asai, T., 1994. Extension of the maximum entropy principle method for directional wave spectrum estimation, in: Proceedings of 24th International Conference Coastal Engineering (ACSCE). Kobe (Japan), pp. 232-246.

Hasselmann, K., Raney, R.K., Plant, W.J., Alpers, W., Shuchman, R.A., Lyzenga, D.R., Rufenach, C.L., Tucker, M.J., Hoogeboom, P., Tucker, M.J., 1985. Theory of Synthetic Aperture Radar Ocean Imaging ' View. J. Geophys. Res. 90, 4659-4686.

Holthuijsen, L.H., 2008. Waves in Oceanic and Coastal Waters. Cambridge University Press, 387 pp.

Huang, W., Carrasco, R., Shen, C., Gill, E.W., Horstmann, J., 2016. Surface Current Measurements Using X-band Marine Radar with Vertical Polarization. IEEE Trans. Geosci. Remote Sens. 54, 2988-2997.

Hwang, P.A., 2008. Observations of swell influence on ocean surface roughness. J. Geophys. Res. 113, 1-14. doi:10.1029/2008JC005075

Hwang, P.A., Wang, D.W., Walsh, E.J., Krabill, W.B., Swift, R.N., 2000. Airborne Measurements of the Wavenumber Spectra of Ocean Surface Waves. Part I: Spectral Slope and Dimensionless Spectral Coefficient. J. Phys. Oceanogr. 2753-2767.

Izquierdo, P., Guedes Soares, C., Nieto Borge, J.C., Rodriguez, G., 2004. A comparison of sea-state parameters from nautical radar images and buoy data. Ocean Eng. 31, 2209-2225.

Janssen, P.A.E.M., 2009. On some consequences of the canonical transformation in the Hamiltonian theory of water waves. J. Fluid Mech. 637, 1-44. doi:10.1017/S0022112009008131

Kleiss, J.M., Melville, W.K., 2010. Observations of Wave Breaking Kinematics in Fetch-Limited Seas. J. Phys 40, 2575-2604. doi:10.1175/2010JPO4383.1

Kudryavtsev, V., Chapron, B., 2016. On Growth Rate of Wind Waves: Impact of Short-Scale Breaking Modulations. J. Phys. Oceanogr. 46, 349-360. doi:10.1175/JPO-D-14-0216.1

Leckler, F., Ardhuin, F., Peureux, C., Benetazzo, A., Bergamasco, F., Dulov, V., 2015. Analysis and interpretation of frequency-wavenumber spectra of young wind waves. J. Phys. Oceanogr. 45, 2484-2496. doi:10.1175/JPO-D-14-0237.1

Longuet-Higgins, M.S., 1963. The effect of nonlinearities on statistical distribution in the theory of sea waves. J. Fluid Mech. 17, 459-480.

Longuet-Higgins, M.S., Cartwright, D.E., Smith, N.D., 1963. Observations of the directional spectrum of sea waves using the motions of a floating buoy., in: International, P.-H. (Ed.), Ocean Wave Spectra. Easton, Maryland (USA), May 1-4, 1961, pp. 111-136.

Ludeno, G., Brandini, C., Lugni, C., Arturi, D., Natale, A., Soldovieri, F., Gozzini, B., Sera, F., 2014. Remocean System for the Detection of the Reflected Waves from the Costa Concordia Ship Wreck. IEEE J. Sel. Top. 
Appl. Earth Obs. Remote Sens. 7, 3011-3018.

Lund, B., Collins, C.O., Graber, H.C., Terrill, E., Herbers, T.H.C., 2014. Marine radar ocean wave retrieval 's dependency on range and azimuth. Ocean Dyn. 64, 999-1018. doi:10.1007/s10236-014-0725-6

Lund, B., Collins, C.O., Tamura, H., Graber, H.C., 2016. Multi-directional wave spectra from marine X-band radar. Ocean Dyn. 66, 973-988. doi:10.1007/s10236-016-0961-z

Nieto Borge, J.C., Rodriguez, G.R., Hessner, K., Gonzalez, P.I., 2004. Inversion of Marine Radar Images for Surface Wave Analysis '. J. Atmos. Ocean. Technol. 21, 1291-1300.

Peureux, C., Benetazzo, A., Ardhuin, F., 2018. Note on the directional properties of meter-scale gravity waves. Ocean Sci. In Press, 1-18. doi:https://doi.org/10.5194/os-2017-48

Phillips, O.M., 1985. Spectral and statistical properties of the equilibrium range in wind-generated gravity waves. J. Fluid Mech. 156, 505-531.

Qiu, J., Zhang, B., Chen, Z., He, Y., 2017. A New Modulation Transfer Function With Range and Azimuth Dependence for Ocean Wave Spectra Retrieval From X-Band Marine Radar Observations. IEEE Geosci. Remote Sens. Lett. 14, 1-5.

Romero, L., Melville, W.K., 2010. Airborne Observations of Fetch-Limited Waves in the Gulf of Tehuantepec. J. Phys. Oceanogr. 40, 441-465. doi:10.1175/2009JPO4127.1

Schwendeman, M., Thomson, J., 2017. Sharp-crested Breaking Surface Waves Observed from a Ship-Based Stereo Video System. J. Phys. Oceanogr. 47, 775-792.

Senet, C.M., Seemann, J., Ziemer, F., 2001. The near-surface current velocity determined from image sequences of the sea surface. IEEE Trans Geosci Remote Sens 39, 492-505.

Serafino, F., Lugni, C., Ludeno, G., Arturi, D., Uttieri, M., Buonocore, B., Zambianchi, E., Budillon, G., Soldovieri, F., 2012. REMOCEAN : A Flexible X-Band Radar System for Sea-State Monitoring and Surface Current Estimation. IEEE Geosci. Remote Sens. Lett. 9, 822-826.

Serafino, F., Lugni, C., Soldovieri, F., 2010. A Novel Strategy for the Surface Current Determination From Marine X-Band Radar Data. IEEE Geosci. Remote Sens. Lett. 7, 231-235.

Sutherland, P., Gascard, J., 2016. Airborne remote sensing of ocean wave directional wavenumber spectra in the marginal ice zone. Geophys. Res. Lett. 43, 5151-5159. doi:10.1002/2016GL067713.1.

Sutherland, P., Melville, W.K., 2013. Field measurements and scaling of ocean surface wave-breaking statistics 40, 3074-3079. doi:10.1002/grl.50584

Young, I.R., Rosenthal, W., Ziemer, F., 1985. A Three-Dimensional Analysis of Marine Radar Images for the Determination of Ocean Wave Directionality and Surface Currents. J. Geophys. Res. 90, 1049-1059.

Zakharov, V.E., Filonenko, N.N., 1967. Energy spectrum for stochastic oscillations of the surface of a liquid. Sov. Phys. Dokl. 11, 881-883.

Zappa, C., Banner, M., Schultz, H., Corrada-Emmanuel, A., Wolff, L., Yalcin, J., 2008. Retrieval of short ocean wave slope using polarimetric imaging. Meas. Sci. Technol. 19, 1-13. 